\title{
Bioética, principios y dilemas éticos en Odontología
}

\author{
Artículo De Revisión
}

\section{Bioethics, principles and ethical dilemmas in dentistry}

\section{Resumen:}

La bioética surge por la necesidad de responder y tratar de solucionar los grandes conflictos que se presentan en la sociedad. La Bioética es un término moderno que se le atribuye la paternidad a Van Rensselaer Potter (1970) al publicar el artículo "Bioethics, the science of survival", Potter establece la existencia de una estrecha relación entre el mundo de las ciencias de la vida, sus hechos y los valores éticos, posteriormente la bioética se relacionó fundamentalmente con la ética médica. Tom L. Beauchamp y James F. Childress publican los "Principios de ética biomédica" el año 1979, siendo considerado un texto clásico en la ética biomédica, en la que se establece cuatro principios bioéticos: la autonomía, no maleficencia, beneficencia y justicia. El análisis y la reflexión bioética son esenciales en el ejercicio profesional del odontólogo, esto permitirá una práctica clínica basado en los valores, que involucrara a todo el personal de salud, orientando las estrategias que permitan afrontar de manera adecuada los dilemas éticos que puedan surgir durante el proceso de atención al paciente. Finalmente, la práctica de odontología requiere de profesionales de un grado más alto en valores.

Palabras claves: Bioética, principios, odontología, dilemas éticos.

\section{Abstracs:}

Bioethics arises from the need to respond and try to solve the major conflicts that arise in society. Bioethics is a modern term that is attributed paternity Van Rensselaer Potter (1970) by publishing the article "Bioethics, the science of survival," Potter establishes the existence of a close relationship between the world of life sciences, his deeds and ethical values, bioethics subsequently primarily it related to medical ethics. Tom L. Beauchamp and James F. Childress published "Principles of biomedical ethics" 1979, being considered a classic text in biomedical ethics, in which four bioethical principles states: autonomy, non-maleficence, beneficence and justice. Analysis and bioethical reflection are essential in the practice of the dentist, this will allow a clinical practice based on values, which would involve all health personnel, guiding strategies to address adequately the ethical dilemmas that may arise during the patient care process. Finally dental practice requires a higher professional level values.

Keywords: Bioethics, principles, dentistry, ethical dilemmas.

\section{Daniel Suárez-Ponce ${ }^{\star} 1$, , Romel \\ Watanabe-Velásquez2, , Sonia Zambrano- De la Peña1,, Abel Anglas-Machacuay2,, Verónica Romero-Álvarez2, Yessica Montano-Rubín De Celis3}

1. Departamento Académico de Estomatología Médico Quirúrgico.

2. Departamento Académico de Estomatología Rehabilitadora.

3. Unidad de Posgrado de la Facultad de Odontología de la Universidad Nacional Mayor de San Marcos.

\section{Correspondencia}

Daniel Guillermo Suárez Ponce*

Correo Electrónico:

daniel06suarez@hotmail.com

Facultad de Odontología de UNMSM. Jr. Amézaga 375, Lima 1, Perú.

Coautores

Watanabe-Velásquez:

rwatanabev@unmsm.edu.pe

Zambrano-De la Peña:

facedent@hotmail.com

Anglas-Machacuay:

abelanglasm@yahoo.es

Romero-Álvarez:

cdveronicaromero@yahoo.com

Montano-Rubín De Celis:

missyessica_1@hotmail.com

Fecha de Recepción : 20/07/2016

Fecha de Aceptación: 31/08/2016

\section{Introducción}

La ética es la filosofía detrás de la moral o la base teórica de la moral. ${ }^{1}$ En el último siglo ha surgido un nuevo movimiento en la ética (la bioética), que trata de unir las ciencias de la vida con varias disciplinas filosóficas, entre ellas la ontología y la ética. ${ }^{1}$

la Bioética, es un término moderno, que es tan antigua como la propia medicina, por ejemplo, el Código de Hammurabi y el Juramento de Hipócrates, incluyen disposiciones relativas a la importancia de las consideraciones éticas en la práctica clínica, además, se centran en las cuestiones éticas relacionadas con la atención clínica. ${ }^{2}$ La bioética se refiere a la moral, al aspecto legal, está relacionado al ámbito político y a los problemas sociales planteados por la medicina, la investigación biomédica, las ciencias de la vida y empleo de las tecnologías. ${ }^{2}$

A pesar del creciente interés, aplicación y evaluación de la bioética en diferentes programas de investigación y procesos normativos, no hay una clara y universal definición de lo que es la bioética. La palabra bioética deriva del griego: bios que significa vida y ethos que significa bueno o malo, correcto o incorrecto. Sobre la base de la ética, la bioética debe hacer frente a los problemas éticos de la vida y también de la muerte, considerando a la muerte como una función de la vida. ${ }^{3}$
La Bioética es la primera y más significativa de las llamadas éticas aplicadas, cuyo origen reciente se sitúa en la segunda mitad del siglo XX, surgiendo como una necesidad social y moral frente a la concepción de la vida y a la acción médica. ${ }^{4}$

En el año 1970, Van Rensselaer Potter (1911-2011), oncólogo de la Universidad de Wisconsin (Madison), publicó el artículo "Bioethics, the science of survival" en la revista Perspectives in Biology and Medicine, la publicación de este artículo le valió la paternidad del término "bioética". 5

El Informe Belmont, publicado en 1978 como resultado de cuatro años de deliberación plural e interdisciplinaria 
de la National Commission for the Protection of Human Subjects of Biomedical and Behavioral Research, establecieron los principios y pautas éticas para la protección de sujetos de investigación y el texto de Beauchamp y Childress publicado en 1979, Principios de ética biomédica, marcaron el inicio de la corriente de resolución de los problemas bioéticos mediante la formulación y aplicación de principios. ${ }^{6}$

La odontología se desarrolla en base a criterios bioéticos, donde el odontólogo debe poseer virtudes morales como la honestidad, la compasión, el altruismo, es alguien de confiar por sus cualidades humanas, por su riqueza espiritual interior. ${ }^{7}$ Además, la reflexión bioética es de vital importancia en la formación del odontólogo, lo que permitirá la apropiación de valores, donde los miembros del equipo de salud bucal tendrán las competencias necesarias para enfrentar los dilemas éticos que puedan surgir en la atención de pacientes odontológicos. ${ }^{8}$

Los conocimientos teóricos del odontólogo deben ser actualizados constantemente, sometiéndose a validación científica en todo momento, esto permitirá que los miembros del equipo de salud bucal reconozcan los riesgos de su entorno y evitaran que ellos provoquen daños a sus pacientes. ${ }^{8}$

Una cuestión ética de otro orden es la resolución de dilemas, esto se produce cuando no vemos clara la conducta a seguir ante una situación conflictiva. La duda que plantea un conflicto ético se puede resolver en ocasiones reflexionando con calma, otras veces consultando con el equipo o recurriendo a procedimientos que sistematizan la toma de decisiones difíciles y, en casos más complejos, consultando a un comité de ética.?

\section{Orígenes de la bioética}

La bioética surge ante la necesidad de responder y tratar de solucionar los grandes conflictos que plantean hoy los hechos científicos y las tecnologías, su uso y aplicación al ser humano, a la vida y a la supervivencia en el planeta. ${ }^{10}$

La bioética fue precedida por la ética médica, que se centraron principalmente en cuestiones que surgen fuera de la relación médico-paciente. ${ }^{11} \mathrm{La}$ antigua literatura de Hipócrates (que incluye pero no se limita con el juramento hipocrático) conmina a los médicos a utilizar sus conocimientos y competencias en beneficio de los enfermos, para sanar y no de causar males, preservando la vida, manteniendo la información en estricta confidencialidad y que no debe ser extendido. ${ }^{11}$ Los valores y principios siguen siendo una parte esencial de la bioética contemporánea. Sin embargo, después de la Segunda Guerra Mundial se hizo evidente que la vieja ética médica no fue suficiente para satisfacer los desafíos contemporáneos. ${ }^{11}$

Van Rensselaer Potter, a finales de 1970, utilizó por primera vez el término "bioética" en su artículo "Bioethics: the science of survival", aunque la divulgación de este neologismo no llegó sino hasta 1971, cuando vio la luz su célebre libro Bioethics: Bridge to the Future $^{12}$, mencionaremos uno de los primeros párrafos de este libro ${ }^{13}$ :

... "El objetivo de este libro es contribuir al futuro de la especie humana al fomentar la formación de una nueva disciplina, la Bioética. Si existen 'dos culturas' que parecen incapaces de hablar una con otra, las ciencias y las humanidades, y si esto constituye una parte de la razón por la cual el futuro parece comprometido, entonces tal vez podríamos construir un 'puente hacia el futuro' construyendo la disciplina de la Bioética como un puente entre las dos culturas. Lo que nos toca afrontar ahora es el hecho de que la ética del ser humano no puede estar separada de una comprensión realista de la ecología en el sentido más amplio. Los valores éticos no pueden estar separados de los hechos biológicos. Tenemos una gran necesidad de una ética de la tierra, de una ética de la vida salvaje, de una ética de las poblaciones, de una ética del consumo, de una ética urbana, de una ética internacional, 'una ética geriátrica', etc. Todas hacen un llamado a acciones basadas en valores y hechos biológicos..." 13.

Como podemos observar en el párrafo anterior Potter visualiza una estrecha relación entre el mundo de las ciencias de la vida, sus hechos y los valores éticos. Comprendía como ciencias de la vida no solamente a las ciencias que tienen que ver con la vida humana, sino también a todas aquellas que engloban su entorno ecológico y ambiental (mundo animal y vegetal). ${ }^{14}$

\section{Principios bioéticos}

La bioética se relacionó especialmente con la ética médica tomando un enfoque direccionado a la investigación médica en humanos. ${ }^{14}$ En 1979 , como una consecuencia del Informe Belmont, Beauchamp y Childress publicaron la primera edición de su libro Principios de Ética Biomédica, estableciendo cuatro principios bioéticos: la autonomía, no maleficencia, beneficencia y justicia, en ediciones recientes el principio de autonomía se describe como el respeto a la autonomía. ${ }^{15}$

Beauchamp y Childress en Principios de ética biomédica, desarrollan de forma amplia loas llamados cuatro principios bioéticos:

- Beneficencia o benevolencia; hacer el bien, velar por la salud de las personas.

- No maleficencia: evitar el mal, el sufrimiento, los perjuicios innecesarios y los riesgos excesivos.

- Justicia: tomar decisiones ecuánimes y actuar con equidad, perspectiva universal.

- Autonomía: respetar los intereses, las preferencias y la capacidad de decisión de cada individuo. ${ }^{16}$

Además, incluyeron reinterpretaciones de los principios tradicionales de la ética médica así como los principios emergentes de los cambios socioculturales a partir del siglo XX.

El principialismo, como su nombre lo indica, utiliza principios éticos como base para tomar decisiones morales. Aplica estos principios a casos o situaciones particulares para determinar qué es lo correcto, tomando en cuenta las reglas y las consecuencias. El principialismo ha influido mucho en debates éticos recientes. ${ }^{16}$

El principialismo ético no implica el uso de una teoría o un modelo formal de decisión; más bien, los principios éticos proporcionan directrices para tomar decisiones morales y justificadas para evaluar idealmente la moralidad de las acciones, cuando se utiliza el enfoque del principialismo, ningún principio es superior a otros principios (Beauchamp y Childress, 2013) y se considera que cada principio es prima facie de unión. ${ }^{16}$ La contribución de Beauchamp y Childress ha sido celebrada como uno de los inventos metodológicos modernos más importantes de ética práctica, donde el principialismo permite localizar los principios morales pertinentes a una situación moral particular y utiliza la especificación, el equilibrio y la aplicación (deductivo) para crear un puente entre la situación moral y los principios pertinentes. ${ }^{17}$

\section{Bioética en odontología}

En vista de la dimensión moral de la conducta del hombre, la Ética y la Bioética, por lo general representan un punto crítico de reflexión sobre la moral, 
por lo que se hace necesario establecer contacto con los valores éticos de una de manera clara, profunda e íntegra, con el fin de cuestionar los valores, con el fin de buscar la consistencia moral. ${ }^{18}$

El análisis y la reflexión ética formal son componentes esenciales en la toma de decisiones de los profesionales de la salud. Así, todas las recomendaciones y procedimientos para la realización de tratamientos tienen una base ética y una consecuencia. Los odontólogos, en cada momento de su vida profesional, se ven enfrentados a conflictos de valores, donde la decisión final afectará el bienestar de sus pacientes de forma importante, estableciéndose una elección moral. ${ }^{19} \mathrm{La}$ compasión, la competencia y la autonomía no son exclusivas a la odontología. Sin embargo, la práctica de odontología requiere profesionales que ejemplifiquen estos valores en un grado más alto que en otras ocupaciones.

\section{Dilemas bioéticos en odontología}

La odontología se encuentra influenciado por cuestiones morales y sociales que han alcanzado a la profesión y han afectado de manera considerable su práctica diaria. En las últimas décadas el equilibrio de la toma de decisiones en la determinación de cuál es el "mejor tratamiento dental" ha desplazado al paternalismo profesional hacia el respeto a la información y autonomía en la decisión del paciente. Los odontólogos han llegado a experimentar problemas cuando los pacientes buscan inapropiados tratamientos, y cuando eligen o se ven limitados a aceptar, el tratamiento por compromiso. ${ }^{20,21}$

La función que debemos cumplir no puede ser al margen de la ética, las decisiones que tomamos tienen un impacto directo en nuestra profesión, por ejemplo si tratamos de obtener beneficios financieros al corto plazo sin tomar en cuenta el aspecto ético en el largo plazo se producirá pérdida de confianza de parte del paciente hacia el odontólogo. ${ }^{20} \mathrm{La}$ búsqueda de nuevos pacientes hace que los profesionales establezcan estrategias de publicidad, distribuyan boletines informativos por correo electrónico a granel, etc., orientado no sólo para retener a los pacientes actuales, sino también para animarles a referir a sus amigos y familias. Además, debemos mencionar que algunos odontólogos al evidenciar la mala calidad trabajo proporcionada por otro profesional, critican abiertamente el trabajo de otros, la cual es contrario a la ética. ${ }^{22} \mathrm{Al}$ referirnos a la ética en el campo odontológico lo que se reclama es la concientización y la responsabilidad moral que entrańa la práctica profesional, el responder cabalmente por su competencia y desempeño profesional, es el requisito indispensable para cumplir con los principios éticos tradicionales: no dañar y hacer el bien. Es preciso, entonces, garantizar la competencia y el desempeño no sólo en los aspectos científicos técnicos, sino también, en lo relacionado con la dimensión humana. ${ }^{23}$

\section{Conclusiones}

1. La reflexión bioética es de vital importancia en el ejercicio profesional del odontólogo, permitiendo una práctica clínica basado en los valores.

2. En nuestra profesión la práctica de la ética influirá en el compromiso por el respeto de las leyes, a las autoridades, el respeto mutuo entre unos y otros y su medio ambiente.

\section{Referencias bibliográficas}

3. Escobar E, Escobar AL. Principales corrientes filosóficas en bioética. Bol. Med. Hosp. Infant. Mex. 2010; 67(3):196-203.

4. Singer P., Viens AM. The Cambridge Textbook of Bioethics. 1a ed. UK: Cambridge University Press; 2008.

5. Nordic Committee on Bioethics. Teaching Bioethics. Reports from a seminar. Copenhagen: Nordic Council of Ministers; 2002.

6. Pérez M. Bioética, Fundamentos, Metodología. Rev. Med. Clin. Condes. 2010; 1(1):130-134.

7. Busquets E. Principios de Ética Biomédica de Tom L. Beauchamp y James F. Childress. Bioética \& Debat. 2011; 17(64):1-7.

8. Escobar J. Riqueza de principios en bioética. Revista Colombiana de Bioética. 2011; 6(2):128-138.

9. Cely G, Herazo B. Bioética para odontólogos. Colombia: Fundación Cultural Javeriana de Artes Gráficas; 2005.

10. Castillo D, Guerra A. Bioética desde una perspectiva odontológica. Rev. Estomat. 2009; 17(2): 45-51.

11. Altisent R. La bioética más allá de los dilemas. Revista Aten. Primaria. 2010;42(8):412-414. doi: $10.1016 /$ j.aprim.2010.01.018
12. Escobar J. Bioética: Origen y tendencias. Rev. Fac. Med. UN Col. 2000; 48(4): 219-223.

13. Steinbock B. The Oxford Handbook of Bioethics. UK: Oxford University Press; 2007.

14. Quintanas A. Reseña de V.R. Potter: Una ética para la vida en la sociedad tecnocientífica. Revista Electrónica Sinéctica. 2009; $32(1): 1-5$.

15. Potter VR. Bioethics: Bridge to the future. USA: Prentice-Hall. 1971.

16. Morales J, Nava G, Esquivel J, Diaz L. Principios de Ética, Bioética y Conocimiento del Hombre. México: Editorial Universitaria UAEH. 2011.

17. Frederick P. Principles of Biomedical Ethics. En: Medical Ethics and Humanities. $1^{\circ}$ ed. USA: Jones and Bartlett Learning; 2009.

18. Christen M. et al. How "moral" are the principles of biomedical ethics? - A cross-domain evaluation of the common morality hypothesis. BMC Medical Ethics. 2014; 15(47): 12pp. Disponible en: http://www.biomedcentral. com/1472-6939/15/47.pdf

19. Rich K. Introduction to bioethics and ethical decision making. En Butts J, Rich K. Nursing Ethics: Across the curriculum and into practice: $4^{\circ}$ ed. USA: Jones and Bartlett Learning; 2016. p. 33-70.

20. Boladeras M. Bioética: Definiciones, prácticas y supuestos antropológicos. Thémata. Revista de Filosofía. 2004; 33: 383-392.

21. Garbin C, Garbin A, Saliba N, Lima D, Macedo A. Analysis of the ethical aspects of profesional confidentiality in dental practice. J Appl Oral Sci. 2008;16(1): 75-80.

22. Williams JR. Dental ethics manual. France: FDI World Dental Federation. 2007.

23. Schwartz B. The ethical implications of patient rewards. J Can Dent Assoc. 2006;72(7): 631-3.

24. Porter SAT, Grey WL. Ethical dilemmas confronting dentists in Queensland, Australia. Australian Dental Journal. 2004;47(3):241-8.

25. Duany T, Turcáz I, González A. Valores éticos en la práctica estomatológica. Compromiso desde la atención primaria. Rev. Med. Electron. 2014;36(6):846-854. 